is, quantitative and is also sensitive to acute changes in free water content without the need for exogenous contrast agents. We hypothesised that non-contrast T1-mapping can serve as a new diagnostic criterion for acute myocarditis.

Methods We studied 23 patients with suspected acute myocarditis and 17 healthy controls. All patients presented with chest pain and troponin I $>0.04 \mathrm{ug} / \mathrm{l}$ and non-obstructive coronary arteries (either on coronary angiogram or ruled out by clinical criteria such as young age or no cardiac risk factors). CMR (at $1.5 \mathrm{~T}$ ) within 10 days included (1) T2-weighted imaging (using the STIR sequence) for oedema; (2) T1-mapping (using the ShMOLLI sequence); and (3) late gadolinium enhancement (LGE) imaging for patterns of cell necrosis (Abstract 091 figure 1). Myocardial T2 signal intensity (SI) relative to skeletal muscle (T2 SI ratio) for detection of oedema and absolute T1 values per-subject were analysed.

Results All patients had a CMR diagnosis of acute myocarditis based on both positive T2-weighted imaging and typical nonischaemic type LGE pattern. Compared to controls, both mean myocardial T1 and T2 SI ratio in patients were significantly higher $(\mathrm{T} 1=1036 \pm 71 \mathrm{~ms}$ vs $\mathrm{T} 1=938 \pm 19$; T2 SI ratio=1.77 \pm 0.24 vs $1.52 \pm 0.10, \mathrm{p}<0.0002$ for both). Receiver operator characteristics analysis showed excellent diagnostic performance for both methods: the area-under-the-curve for T1-mapping $=0.96$ and T2-weighted imaging=0.93 ( $p=0.3$, Abstract 091 figure 2). At a T1 value of $958 \mathrm{~ms}$, the sensitivity and specificity were $87 \%$.

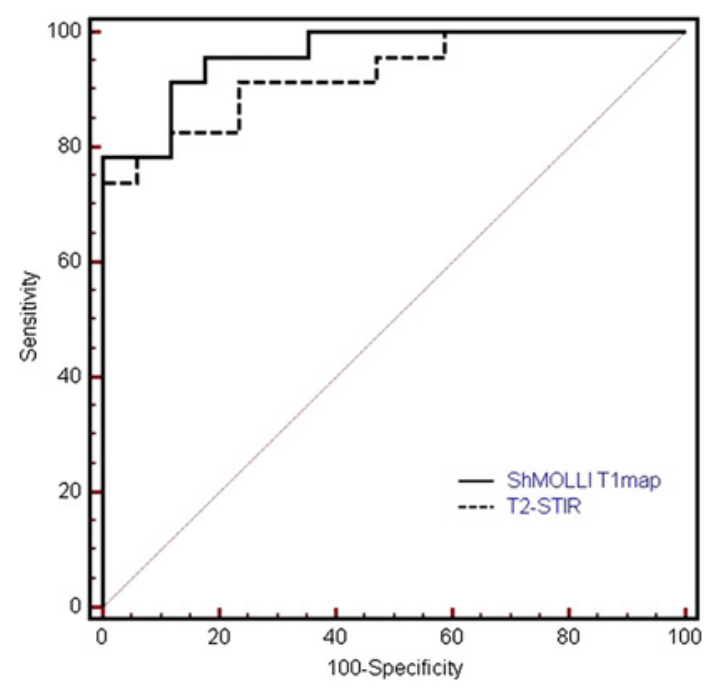

Abstract 091 Figure 2 ROC curves for ShMOLLI T1-mapping and T2-STIR in acute myocarditis.

Conclusions Non-contrast T1-mapping has a high diagnostic performance for acute myocarditis and may be used as a novel additional CMR diagnostic criterion.

\section{INTERSTITIAL EXPANSION IN HEALTH AND DISEASE-AN EQUILIBRIUM CONTRAST CMR STUDY}

doi:10.1136/heartjnl-2012-301877b.92

${ }^{1} \mathrm{D}$ M Sado, ${ }^{*} \mathrm{~A}$ S Flett, ${ }^{1} \mathrm{~S}$ Banypersad, ${ }^{1} \mathrm{~S}$ White, ${ }^{2} \mathrm{D}$ Hughes, ${ }^{2} \mathrm{~A}$ Mehta, ${ }^{3} \mathrm{E}$ Murphy, ${ }^{3} \mathrm{R}$ Lachmann, ${ }^{2} \mathrm{P}$ Hawkins, ${ }^{1} \mathrm{D}$ Hausenloy, ${ }^{1} \mathrm{~W}$ McKenna, ${ }^{4} \mathrm{~A}$ Taylor, ${ }^{1} \mathrm{P}$ Elliott, ${ }^{1} \mathrm{~J}$ C Moon. ${ }^{1} T h e$ Heart Hospital, London, UK; ${ }^{2}$ The Royal Free Hospital, London, UK; ${ }^{3}$ Oueens Square Hospital, London, UK; ${ }^{4}$ Great Ormond Street Hospital, London, UK

Introduction Interstitial myocardial volume expansion is an important factor in cardiac disease but until recently could only be accurately assessed with myocardial biopsy. It is usually a result of diffuse fibrosis, but can also be caused by infiltration (such as by amyloid deposition) or oedema. We have used a new method,
Equilibrium Contrast Cardiovascular Magnetic Resonance (EOCMR) to accurately quantify the interstitial space in normal subjects and across a broad spectrum of cardiac diseases.

Methods The three steps in EQ-CMR are: (1) a primed gadolinium infusion to achieve contrast equilibrium, (2) Signal (T1) measurement pre and post equilibrium, (3) measurement of blood contrast volume (1-haematocrit). This allows calculation of the contrast volume of distribution, $\mathrm{Vd}(\mathrm{m})$, by:

$\mathrm{Vd}(\mathrm{m})=(1-$ haematocrit $) \times \Delta(1 / \mathrm{T} 1)$ myo $\div \Delta(1 / \mathrm{T} 1)$ blood

$\mathrm{Vd}(\mathrm{m})$ was measured in 278 subjects: 86 normal subjects (median age 43, range 24-81, 51\% male) and 192 patients with AndersonFabry disease (AFD, $n=17$ ), dilated cardiomyopathy (DCM, $n=31$ ), hypertrophic cardiomyopathy (HCM, $n=31$ ), severe aortic stenosis (AS, $n=66)$, cardiac AL amyloidosis $(n=27)$ or myocardial infarction (MI, $\mathrm{n}=20$ ).

Results In normal subjects, mean $\mathrm{Vd}(\mathrm{m})$ was higher in females (0.274) than males $(0.237, \mathrm{p}<0.001)$. In all diseases, $\mathrm{Vd}(\mathrm{m})$ was higher than normal subjects $(p<0.001)$ except the intracellular storage disease AFD $(0.250, p=0.9) . \mathrm{Vd}(\mathrm{m})$ was the same in DCM (0.280), HCM (0.291) and AS (0.276), but higher in the exemplar of infiltrative disease, cardiac AL amyloidosis (0.466) and higher again in MI (0.585, each $p<0.001)$, Abstract 092 figure 1 . These trends were also present when disease data were compared to gender matched normal subjects. Where $\mathrm{Vd}(\mathrm{m})$ was elevated, correlations existed with important clinical CMR parameters including ejection fraction, indexed left ventricular mass, end systolic volume and left atrial area, in apparent disease specific patterns, Abstract 092 table 1.

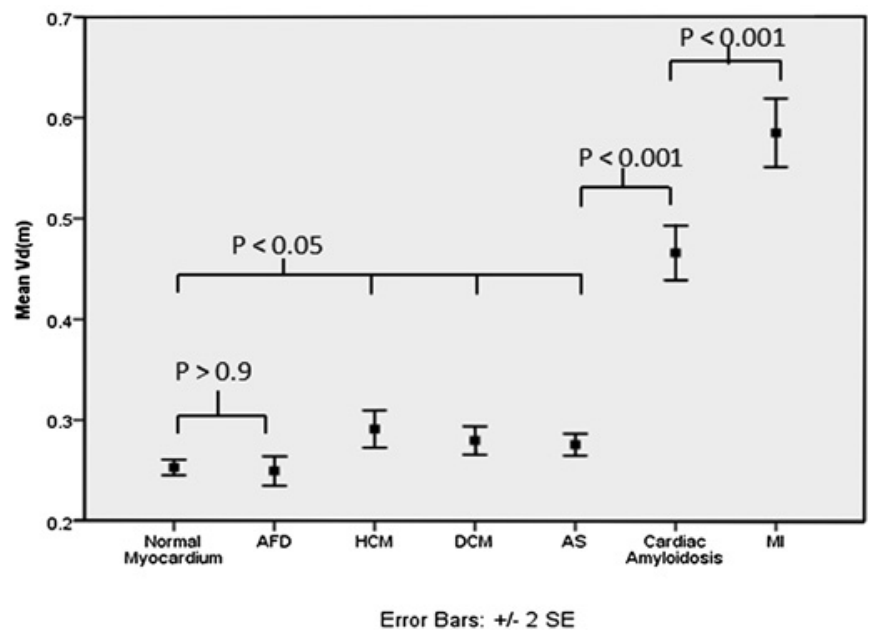

Abstract 092 Figure 1

Abstract 092 Table 1 Correlations assessed using Pearson coefficient

\begin{tabular}{lllll}
\hline Disease & $\begin{array}{l}\text { Ejection } \\
\text { fraction }\end{array}$ & $\begin{array}{l}\text { Indexed end } \\
\text { systolic volume }\end{array}$ & $\begin{array}{l}\text { Indexed left } \\
\text { ventricular mass }\end{array}$ & $\begin{array}{l}\text { Indexed left } \\
\text { atrial area }\end{array}$ \\
\hline Normal subjects & No correlation & No correlation & $\mathrm{R}=-0.36^{* *}$ & No correlation \\
AFD & No correlation & No correlation & No correlation & No correlation \\
DCM & $\mathrm{R}=-0.35^{*}$ & No correlation & $\mathrm{R}=-0.36^{* *}$ & $\mathrm{R}=0.65^{* * *}$ \\
AS & No correlation & $\mathrm{R}=0.51^{*}$ & No correlation & No correlation \\
HCM & No correlation & No correlation & No correlation & No correlation \\
Cardiac AL & $\mathrm{R}=-0.57^{* *}$ & $\mathrm{R}=0.63^{* * *}$ & $\mathrm{R}=0.44^{*}$ & No correlation \\
amyloidosis & & & & \\
\hline${ }^{*} \mathrm{p}<0.05,{ }^{* *} \mathrm{p}<0.01,{ }^{* * *} \mathrm{p}<0.001$. & &
\end{tabular}

Conclusions This study shows the ability of EQ-CMR to noninvasively quantify interstitial expansion using this novel technique in both the healthy population and a spectrum of differing cardiac 
diseases. Further work will build on this study to evaluate the potential role of $\mathrm{Vd}(\mathrm{m})$ as a clinical biomarker.

\section{CARDIAC INVOLVEMENT IN CARDIAC AL AMYLOIDOSIS AS MEASURED BY EQUILIBRIUM CONTRAST CARDIOVASCULAR MAGNETIC RESONANCE}

doi:10.1136/heartjnl-2012-301877b.93

${ }^{1} S$ M Banypersad, ${ }^{*} \mathrm{D}$ Sado, ${ }^{1} \mathrm{~A} S \mathrm{~S}$ Flett, ${ }^{2} \mathrm{~S}$ Gibbs, ${ }^{2} \mathrm{~J}$ Pinney, ${ }^{1} \mathrm{~V}$ Maestrini, ${ }^{2} \mathrm{~A}$ Wechalekar, ${ }^{2} \mathrm{P}$ N Hawkins, ${ }^{1} \mathrm{~J} \mathrm{C}$ Moon. ${ }^{1}$ The Heart Hospital, London, UK; ${ }^{2}$ National Amyloidosis Centre, London, UK

Introduction Cardiac involvement drives prognosis in Systemic AL Amyloidosis, predicting outcome and influencing therapeutic options. Current methods of cardiac assessment do not quantify the myocardial amyloid burden. We used Equilibrium Contrast Cardiovascular Magnetic Resonance (EQ-CMR) to measure the cardiac interstitial compartment, measured as the myocardial contrast volume of distribution, $\mathrm{Vd}_{\mathrm{m}}$, which we hypothesised would reflect the amyloid burden.
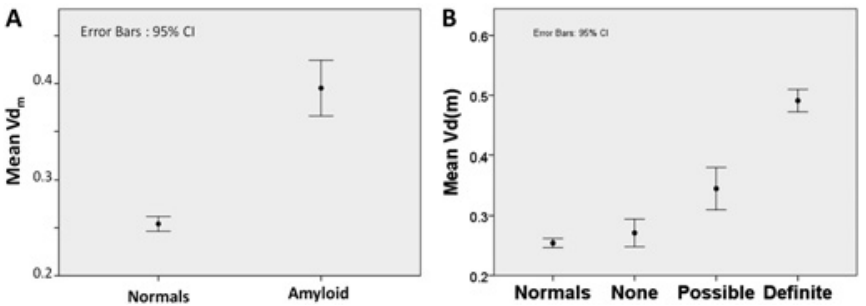

Abstract 093 Figure 1

Methods Patients with systemic AL amyloidosis undergoing routine work up at the National Amyloidosis Centre were recruited $(n=60$, 39 males, 21 females, mean age 63 years) and underwent conventional CMR including late enhancement, EQ-CMR to measure $\mathrm{Vd}_{\mathrm{m}}$ and standard cardiac work-up including ECG, echocardiography, biomarkers (BNP, Troponin T) and functional assessment (6-min walk test, 6MWT, where permitted by autonomic neuropathy). Results were compared to normal controls. Conventional assessment ranked cardiac involvement as definite, probable and none.

Results $\mathrm{Vd}_{\mathrm{m}}$ was significantly higher in patients than normal controls ( 0.25 vs $0.40, p<0.001)$ (see Abstract 093 figure $1 \mathrm{~A})$. This tracked conventional cardiac assessment (none, probable, definite corresponded with a $\mathrm{Vd}_{\mathrm{m}}$ of 0.276 vs 0.342 vs $0.488, \mathrm{p}<0.005$ ), respectively (see Abstract 093 figure $1 \mathrm{~B}$ ). $\mathrm{Vd}_{\mathrm{m}}$ correlated with cardiac parameters by echo (eg, TDI S-wave $\mathrm{R}^{2} 0.27, \mathrm{p}<0.001$ ) and
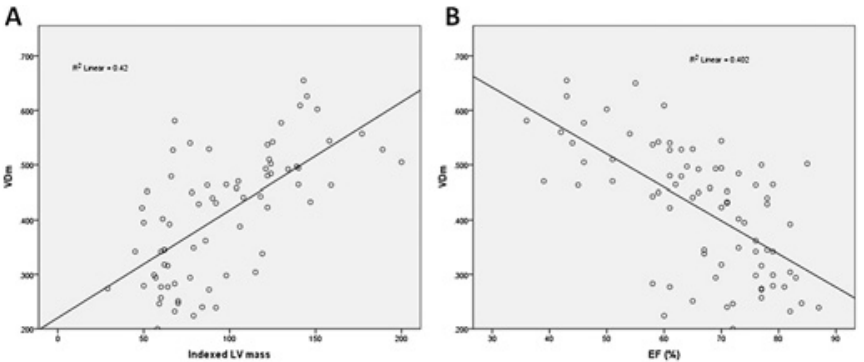

Abstract 093 Figure 2 conventional CMR (eg, indexed LV mass $R^{2} 0.31, p<0.001$ - see Abstract 093 figure 2). Significant correlations were also seen with BNP $\left(R^{2} 0.47, p<0.001\right)$ and Troponin $T\left(R^{2} 0.28, p=0.006\right) . V_{m}$ was associated with ECG abnormalities and tracked small ORS voltages $\left(\mathrm{R}^{2}\right.$ 0.33, $\left.\mathrm{p}<0.001\right)$. A higher $\mathrm{Vd}_{\mathrm{m}}$ correlated with a lower 6MWT outcome $\left(\mathrm{R}^{2} 0.13, \mathrm{p}=0.03\right)$.

Conclusions The measurement of the myocardial interstitial compartment $\left(\mathrm{Vd}_{\mathrm{m}}\right)$ using EQ-CMR in systemic $\mathrm{AL}$ amyloidosis quantifies the cardiac amyloid burden.

\section{THE PREVALENCE OF NON-CALCIFIED PLAQUES IN SYMPTOMATIC PATIENTS WITH ZERO CALCIUM SCORE}

doi:10.1136/heartjnl-2012-301877b.94

${ }^{1} \mathrm{G}$ K Koulaouzidis, ${ }^{*} \mathrm{D}$ C Charisopoulou, ${ }^{2} \mathrm{~T} \mathrm{M}$ McArthur, ${ }^{2} \mathrm{~S} M$ Maffrett, ${ }^{2} \mathrm{M} \mathrm{T}$ Tighe ${ }^{2} \mathrm{P} J \mathrm{~J}$ Jenkins. ${ }^{1}$ Heart Centre and Department of Public Health and Clinical Medicine, Umea University, Umea, Sweden; ${ }^{2}$ European Scanning Centre, London, UK

Introduction Atheromatous plaque rupture is the most common cause of coronary artery thrombosis. Non-calcified plaques, with thin fibrous cap and large thrombogenic lipid core, are predominantly the most susceptible to rupture.

Aims To investigate, with a 640-slice, 320-row CT scanner, the noncalcified coronary artery plaques (NCAP) prevalence and the degree of caused obstruction, in a cohort of symptomatic subjects, without coronary calcification.

Methods and Results Out of 1806 patients, who underwent coronary CT angiography (CTCA), we retrospectively identified 447 symptomatic patients with coronary artery calcification (CAC) score of 0 . Standard cardiovascular risk factors were assessed prior to the CTCA study. From the 447 subjects, 400 (89.48\%) had a negative CTCA, while in $47(10.51 \%)$ NCAP were depicted on CTCA. Four of these (4/47) had stenosis more than $50 \%$. Mean age of patients with positive CTCA was 56.21 years, significantly higher than those of patients with negative CTCA (50.6 years, $p<0.004$ ) Additionally, when compared to patients with normal CTCA, those with NCAP were in higher risk of developing CAD, as derived from the pre CTCA assessment ( $26 \%$ vs $34.04 \%, p<0.0001)$. The Left Anterior Descending artery (LAD), and especially the proximal segment, was the predominant location for the development of NCAP.

Conclusion Absence of coronary calcification does not exclude the presence of atherosclerosis; NCAP is present in up to $10 \%$ of patients with CAC score of 0 . Symptomatic patients, who older in age, with multiple factors and high probability of CAD, would benefit from CTCA even in the absence of CAC.

\section{PLAQUE MAPPING BASED ON CONTRAST RATIOS PERMITS IDENTIFICATION OF UNSTABLE CORONARY PLAQUE AND QUANTIFICATION OF CORONARY ATHEROSCLEROSIS BY CORONARY CT ANGIOGRAPHY}

doi:10.1136/heartjnl-2012-301877b.95

${ }^{1} \mathrm{D}$ R Obaid, ${ }^{*}{ }^{1} \mathrm{P}$ A Calvert, ${ }^{2} \mathrm{M}$ Goddard, ${ }^{2} \mathrm{D}$ Gopalan, ${ }^{1} \mathrm{~J} \mathrm{H}$ F Rudd, ${ }^{1} \mathrm{M}$ R Bennett. ${ }^{1}$ University of Cambridge, Cambridge, UK; ${ }^{2}$ Papworth NHS Foundation Trust Hospital, Cambridge, UK

Background Previous attempts to characterise coronary components using CT have relied on fixed Hounsfield unit (HU) ranges which do not correct for the effect of inter-patient variation of contrast intensity on plaque attenuation. We examine the utility of using HU-ranges derived from contrast attenuation ratios.

Methods 57 patients underwent coronary CT and Virtual Histology IVUS examination. Attenuation was sampled in over 1000 plaque 